\title{
Prevalencia de Neisseria gonorrhoeae, en reclusos del Centro de Detención Preventiva de Arica
}

\section{Prevalence of Neisseria gonorrhoeae, amongst inmates of the Preventive Reclusion Center in Arica}

\author{
Teresa Reyes R. ${ }^{1}$, Hilda Villanueva', Celia Bórquez B. ${ }^{2}$, Dayana Casanova B. ${ }^{2}$, Valeska Hahn A. ${ }^{2}$, Diego Matienzo S. ${ }^{2}$, \\ Camila Villalobos $R^{2}$ y Juan Vega ${ }^{3}$
}

'Facultad de Ciencias de la Salud, Departamento de Obstetricia. Universidad de Tarapacá. Arica, Chile.
2Facultad de Ciencias de la Salud, Departamento de Tecnología Médica. Universidad de Tarapacá. Arica, Chile.
${ }^{3}$ Facultad de Ingeniería. Universidad de Tarapacá. Arica, Chile.

No existen conflictos de interés para este estudio inédito.

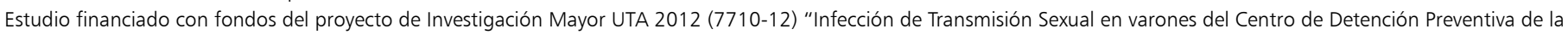
Región de Arica-Parinacota".

Recibido: 20 de diciembre de 2018 / Aceptado: 30 de diciembre de 2019

\section{Resumen}

Introducción: Los/as trabajadores/as sexuales, personas con adicción a drogas, población de inicio sexual precoz y población penal son considerados los grupos de mayor riesgo de contraer infecciones de transmisión sexual (ITS). Objetivo: Determinar prevalencia de infección por Neisseria gonorrhoeae, en reclusos del Centro de Detención Preventiva (CDP) de la Región de Arica y Parinacota, Chile. Este estudio contó con la aprobación del Cómité Ético Científico de la Universidad de Tarapacá. Material y Método: Participaron 140 reclusos, que aceptaron ser parte del estudio en forma voluntaria y firmaron un consentimiento informado. Se tomó una muestra del meato uretral para pesquisa de $N$. gonorrhoeae y se aplicó una encuesta epidemiológica que consignó edad, consumo de drogas, hacinamiento, entre otros. Resultados: La prevalencia del agente fue de $16,4 \%$ en reclusos del CDP de Arica, resultado menor a lo reportado en otros estudios similares. Conclusiones: Conocer la realidad de la prevalencia de esta ITS y algunos factores de riesgo asociados a la situación de privación de la libertad en una zona tri-fronteriza del norte de Chile, contribuye a las propuestas de programas de prevención en esta población vulnerable y de riesgo.

Palabras clave: Hombres en reclusión; Neisseria gonorrhoeae; gonorrea; cárcel.

\begin{abstract}
Background: Sex workers, people with drug addiction, early onset of sexual activity population, and criminal population, are considered the groups most at risk of contracting sexually transmitted infections (STIs). Aim: To determine the prevalence of infection by Neisseria gonorrhoeae in inmates of the Preventive Detention Center (CDP) at Arica and Parinacota Region, Chile. The Scientific Ethical Committee of Universidad de Tarapacá approved this study. Method: 140 inmates participated, who voluntarily agreed to be part of the study and signed an informed consent. A sample of urethral meatus was taken to investigate $N$. gonorrhoeae, and an epidemiological survey was applied, which included age, drug use, overcrowding, among others. Results: The prevalence of the agent was $16.4 \%$ in inmates of the Arica CDP, a result lower than that reported in other similar studies. Conclusion: Knowing the reality of the prevalence of this STI and some risk factors associated with the situation of deprivation of freedom in a tri-border area of northern Chile, contributes to the proposals for prevention programs in this vulnerable and at-risk population.

Keywords: Men in confinement; Neisseria gonorrhoeae; gonorrhea; penitentiary.
\end{abstract}




\section{Introducción}

as infecciones de transmisión sexual (ITS) siguen siendo en la actualidad un problema importante de salud pública en el mundo ${ }^{1}$. Comprenden un grupo de patologías de etiología infecciosa diversa, que se transmiten generalmente por contacto sexual ${ }^{2,3}$, y se encuentran distribuidas ampliamente en el mundo.

La Organización Mundial de la Salud (OMS) estima que más de 1 millón de personas contraen diariamente una ITS y que anualmente unos 357 millones de personas contraen alguna de las cuatro ITS curables de mayor prevalencia: infección por clamidia (131 millones), gonorrea (78 millones), sífilis (5,6 millones) y tricomoniasis (143 millones) $)^{2,3}$.

Neisseria gonorrhoeae muestra en Chile una tasa en descenso desde 1990 a 1996. En los últimos años (desde el 2006) hay una tendencia estacionaria con un leve repunte en el $2010^{4}$. Se observa un aumento en el número de cepas confirmadas de $N$. gonorrhoeae entre los años 2010 y 2015. El año 2015 se confirmó el mayor número de casos, con un aumento de $208,3 \%$ en relación al año $2010^{5}$. El informe anual 2016 de Cáceres K. sobre situación epidemiológica de gonorrea en Chile, refiere un incremento de $38 \%$ en relación al quinquenio anterior ${ }^{6}$.

Diversos estudios han reportado que los grupos con mayor riesgo de contraer una ITS, son las trabajadoras sexuales, las personas privadas de libertad, y en la población mundial, los adolescentes, los jóvenes y las mujeres.

La población penal presenta alto riesgo de adquirir y transmitir una ITS, dado las condiciones socio-demográficas en las cuales conviven los internos de los recintos penitenciarios, donde el hacinamiento, la infraestructura inadecuada, carencia de luz natural, ventilación, higiene y aseo, junto a la condición de alta vulnerabilidad, prácticas sexuales y falta de educación propician estas infecciones ${ }^{7-9}$.

Considerando que la mayoría de la ITS se presentan de manera asintomática en los hombres y no son tratadas de manera oportuna, se vuelven un riesgo latente para su transmisión, tanto en el recinto que los alberga como en la comunidad.

El Centro de Detención Preventiva (CDP) de la Región de Arica y Parinacota, ubicada en la entrada sur de la ciudad de Arica, está organizada en tres sub-sistemas penitenciarios, en los cuales se divide la población penal (cerrado, semi abierto y abierto), posee una capacidad -total para 1.100 reclusos y en el periodo de estudio esta población fue de 2.394, con un índice de sobrepoblación de $118 \%{ }^{10}$. Este recinto fue construido bajo el modelo de celdas individuales; sin embargo, son ocupadas generalmente por dos o más individuos ${ }^{11}$.
El objetivo de este estudio fue, determinar la prevalencia de la infección por $N$. gonorrhoeae y factores de riesgo asociados, en los internos del CDP de Arica y Parinacota, como grupo poblacional con características socio-culturales definidas y factores de riesgo que le otorgan una mayor vulnerabilidad. El estudio pretende aportar información al respecto, dado que en Chile son escasos los trabajos sobre este tema.

\section{Material y Método}

Se realizó un estudio descriptivo, de corte transversal, en el CDP de la Región de Arica y Parinacota, Chile. Para la selección de la muestra los criterios de inclusión fueron ser recluso de sexo masculino, imputado, y ser usuario del Centro de Salud del penal.

La población que cumplió con estos criterios correspondió a 483 reclusos, de éstos, 140 individuos conformaron la muestra, cuyas edades fluctuaban entre 19 y 77 años de edad, asintomáticos y sintomáticos y fueron atendidos entre los meses de junio - diciembre de 2013. Participaron en forma voluntaria, previo conocimiento y firma del consentimiento informado. La selección de la muestra no fue aleatoria, por lo tanto, tendrá limitaciones propias de una muestra no probabilística.

Cada individuo de la muestra respondió una encuesta epidemiológica focalizada en los objetivos del estudio, recogiendo datos referidos a edad, conductas de riesgo para adquirir ITS (orientación sexual, consumo de drogas, edad de inicio de relaciones sexuales) y otras variables relacionadas con la permanencia en prisión y grado de hacinamiento.

Se tomó una muestra uretral con tórula estéril para la pesquisa de N. gonorrhoeae. Una vez obtenida la muestra, fue sembrada de inmediato en medio ThayerMartin, utilizado para el aislamiento de $N$. gonorrhoeae, el cual contiene enriquecedores y una mezcla selectiva de agentes antimicrobianos que inhiben la microbiota acompañante para permitir un buen desarrollo de esta bacteria altamente exigente. Luego fue realizado un extendido de la secreción en un portaobjetos y tinción de Gram, para la demostración al microscopio óptico de diplococos gramnegativos intra y extracelulares. En aquellos individuos que no presentaban secreción uretral, se solicitó exprimir la uretra desde atrás hacia delante para lograr el exudado, pero aún así, en alguno de éstos no se logró, por lo que se debió introducir la tórula en forma suave al meato uretral girándola durante 5 segundos.

Las muestras fueron transportadas al laboratorio de Microbiología de la Universidad de Tarapacá, en caja de bioseguridad para el correspondiente procesamiento. 


\begin{tabular}{lcc|}
\hline \multicolumn{3}{l|}{$\begin{array}{l}\text { Tabla 1. Prevalencia de infección por Neisseria } \\
\text { gonorrhoeae en reclusos del CDP de Arica }\end{array}$} \\
\hline Agente ITS & $\mathbf{n}$ & Prevalencia (\%) \\
N. gonorrhoeae & 23 & 16,4 \\
Negativo & 117 & 83,6 \\
Total & 140 & 100 \\
\hline
\end{tabular}

\section{Análisis estadístico}

Los datos se analizaron descriptivamente y para determinar la asociación se aplicó $\chi^{2}$, utilizando el programa estadístico SPSS v.23.

\section{Resultados}

De los 140 individuos estudiados, la prevalencia de $N$. gonorrhoeae fue de $16,4 \%$ con 23 casos positivos (Tabla 1).

La edad mínima fue de 19 años, la máxima de 77 años correspondiendo la edad media a 36,7 años con una desviación estándar de 12,1 años (Tabla 2). La mayor frecuencia de $N$. gonorrhoeae se presentó en individuos en el rango etario de 19-30 años $(8,6 \%)$ seguida por el grupo de 31-40 años (5,0\%). No existió asociación estadística entre grupo etario e infección por $N$. gonorrhoeae $(\mathrm{p}=0,303)$.

El inicio de actividad sexual del total de los individuos positivos fue 18 años y menos, correspondiendo una prevalencia de $9,3 \%(\mathrm{n}=13)$ al grupo de 14 y menos años (Tabla 3). No existió asociación estadística entre edad de inicio de relaciones sexuales y la portación de la ITS estudiada $(\mathrm{p}=0,115)$.

Según el número de reclusos por celda (hacinamiento) y presencia de la ITS estudiada (Tabla 4), se observó mayor prevalencia de $N$. gonorrhoeae en aquellos que compartían ese espacio, con 8,6\% $(\mathrm{n}=12)$ en celdas con 2 usuarios y $6,4 \%(n=9)$ en celdas con 3 o más usuarios. No se comprobó asociación estadística entre el nivel de hacinamiento de los reclusos y $N$. gonorrhoeae al 5\% $(\mathrm{p}=0,159)$.

El $76,4 \%(n=107)$ de los internos reconoció el consumo de drogas (Tabla 5) y una prevalencia de $15,7 \%$ $(\mathrm{n}=22)$ en los reclusos con infección por N. gonorrhoeae, encontrándose que existe asociación estadística entre consumo de drogas e infección por $N$. gonorrhoeae al $5 \%(\mathrm{p}=0,018)$.

De acuerdo a la orientación sexual de los internos (Tabla 6), el mayor porcentaje de ITS se observó en individuos heterosexuales correspondiendo a $12,9 \%$ (n: 18), con una asociación baja.
Tabla 2. Prevalencia de Neisseria gonorrhoeae de acuerdo al grupo etario en reclusos del CDP de Arica

\begin{tabular}{ccccc}
$\begin{array}{c}\text { Grupo etario } \\
\text { (años) }\end{array}$ & $\mathbf{n}$ & Total & $\%$ & \multicolumn{2}{c}{ N. gonorrhoeae } \\
$19-30$ & 58 & 41,4 & 12 & $\%$ \\
$31-40$ & 36 & 25,7 & 7 & 8,6 \\
$41-50$ & 23 & 16,4 & 3 & 2,0 \\
$\geq 51$ & 23 & 16,4 & 1 & 0,7 \\
Total & 140 & 100 & 23 & 16,4 \\
\hline 0,303$).$ & & & & \\
\hline
\end{tabular}

Tabla 3. Prevalencia de Neisseria gonorrhoeae según la edad de inicio de relaciones sexuales en reclusos del CDP de Arica

\begin{tabular}{cccccc}
\hline $\begin{array}{c}\text { Edad de inicio de la } \\
\text { actividad sexual (años) }\end{array}$ & $\mathbf{n}$ & Total & $\%$ & \multicolumn{2}{c}{ N. gonorrhoeae } \\
\hline $10-14$ años & 57 & 40,7 & 13 & 9,3 \\
$15-18$ años & 71 & 50,7 & 10 & 7,1 \\
$19-22$ años & 12 & 8,6 & 0 & 0 \\
\hline Total & 140 & 100,0 & 23 & 16,4 \\
\hline$(p=0,115)$. & & & \\
\hline
\end{tabular}

Tabla 4. Prevalencia de Neisseria gonorrhoeae según grado de hacinamiento en reclusos del CDP de Arica

\begin{tabular}{cccccc}
\hline $\begin{array}{c}\text { Hacinamiento (n de personas } \\
\text { por celda individual) }\end{array}$ & $\mathbf{n}$ & Total & \% & \multicolumn{2}{c}{$\boldsymbol{N . \text { gonorrhoeae }}$} \\
$\mathbf{n}$ & 38 & 27,1 & 2 & 1,4 \\
2 & 56 & 40,0 & 12 & 8,6 \\
$\geq 3$ & 46 & 32,9 & 9 & 6,4 \\
Total & 140 & 100 & 23 & 16,4 \\
\hline$(p=0,159)$. & & & & \\
\hline
\end{tabular}

Tabla 5. Prevalencia de portadores de ITS de acuerdo al consumo de drogas en reclusos del CDP de Arica

\begin{tabular}{cccccc}
\hline Consumo de drogas & & Total & \multicolumn{2}{c}{$\boldsymbol{N}$. gonorrhoeae } \\
& $\mathbf{n}$ & & $\%$ & $\mathbf{n}$ & $\%$ \\
Sí & 107 & 76,4 & 22 & 15,7 \\
No & 33 & 23,6 & 1 & 7,1 \\
Total & 140 & 100,0 & 23 & 16,4 \\
\hline$(p=0,018)$. & & & \\
\hline
\end{tabular}

Tabla 6. Prevalencia de ITS según orientación sexual de los internos en reclusos del CDP de Arica

\begin{tabular}{lccccc}
\hline Orientación sexual & Total & \multicolumn{2}{c}{ N. gonorrhoeae } \\
& $\mathbf{n}$ & $\mathbf{\%}$ & $\mathbf{n}$ & $\%$ \\
Heterosexual & 119 & 90,2 & 18 & 12,9 \\
Bisexual & 8 & 6,1 & 4 & 2,9 \\
Homosexual & 5 & 3,8 & 1 & 0,7 \\
Total & 132 & 100 & 23 & 16,4 \\
\hline$(p=0,040)$. & & & \\
\hline
\end{tabular}




\section{Discusión}

De acuerdo al informe emitido por el Boletín de Vigilancia del Instituto de Salud Pública (ISP), en el país la gonorrea ha aumentado $208 \%$ en cinco años. El informe describe que en el año 2015 se detectó el mayor número de casos (1.640) con una tasa de 10 casos de gonorrea por cien mil habitantes ${ }^{5}$. En el presente estudio la prevalencia fue de 16,4\% en reclusos del CDP de Arica y Parinacota, Chile. La prevalencia de ITS -sólo se evaluó gonorrea- en población privada de libertad del CDP de Arica es menor a lo reportado en el estudio realizado a internos de la cárcel de Daroca, en Zaragoza España, que presenta una prevalencia de ITS de $38,7 \%$ y es superior al compararlo con mujeres privadas en libertad en Lima y Estados Unidos de América ${ }^{8,12,13}$.

En relación a la distribución por sexo, según lo reportado por el ISP para el período $2010-2016,90,2 \%$ de las cepas confirmadas provenían de pacientes varones. Esta cifra se explica debido a que la presentación clínica es sintomática en los hombres, lo que facilita la consulta y tratamiento oportuno ${ }^{5}$. El resultado obtenido también resultó mayor a lo reportado en población general en Perú ${ }^{12}$ y en el estudio realizado en Mar del Plata, en el que se obtuvo una frecuencia de infección por $N$. gonorrhoeae de $12,2 \%$, en el total de las muestras procesadas, resultando significativamente más frecuentes en los consultantes masculinos que en las mujeres ${ }^{14}$.

Con respecto a la edad de los individuos en estudio, $82,6 \%$ de los infectados por $N$. gonorrhoeae fueron menores de 41 años. Destaca especialmente el grupo bajo 31 años de edad, donde se encontró la mayor prevalencia $(52,1 \%)$, coincidiendo con la edad de mayor actividad sexual. El MINSAL reportó en el año 2010, que 63,6\% de los casos positivos se concentró entre los 15 y 29 años de edad ${ }^{4}$.

El $91,3 \%$ de los reclusos portadores se encontraban en situación de hacinamiento, conviviendo dos o más personas en una habitación al momento del estudio. Estos resultados son comparables con los hallazgos descritos para recintos penitenciaros en Chile y el extranjero ${ }^{15-17}$. La condición de hacinamiento es un ambiente epidemiológico favorable para contraer ITS en la población general y cobra mayor importancia en la población penal, en Chile la ocupabilidad de la celda supera el $25 \%$, siendo mayor en centros penitenciarios en España $a^{18,19}$.

Se ha demostrado que mientras más precoz es el inicio de las relaciones sexuales, aumenta el número de parejas sexuales y el riesgo de contraer y transmitir una ITS ${ }^{19}$. En Chile según la VIII Encuesta Nacional de la Juventud, la edad de inicio de la actividad sexual es de 16,3 años en los hombres ${ }^{20}$. Los resultados obtenidos mostraron una moderada asociación entre la edad de inicio de la actividad sexual y la infección por $N$. gonorrhoeae. El total de los infectados inició la actividad sexual antes de los 19 años, destacando que 56,5\% $(\mathrm{n}=13)$ la inició antes de los 14 años. En un estudio en el mismo centro penitenciario de Arica, respecto a otras ITS, refieren que la mayor prevalencia de ITS se observó en el grupo que inició la actividad sexual antes de los 19 años $(92,1 \%)^{21}$.

En tanto, el consumo de drogas y alcohol en base a lo reportado por el estudio "Condiciones de vida en los centros privados de libertad en Chile"17, informa que $20 \%$ de los encuestados posee una adicción al alcohol o a algún tipo de droga, la que sería una de las más altas de los países de la región; esta cifra no concuerda con lo reportado por el presente estudio, en que $76,4 \%$ $(n=107)$ declara ser consumidor de droga, muy mayor a lo reportado en una población similar en España que describe $23,1 \%$ de consumo de droga ${ }^{22}$. De igual manera, este alto consumo revela un grave problema relacionado con seguridad, corrupción y la presencia de ITS ya que 95,6\% de los infectados con $N$. gonorrhoeae informó consumo de drogas.

Según la orientación sexual de los portadores de ITS, $78,2 \%$ se declaró heterosexual, mientras que 17,4 y 4,3\% se declaró bisexual y homosexual, respectivamente, proporción semejante referida en el estudio realizado a internos de diferentes CDP en Santiago de Chile ${ }^{23}$. Al analizar la prevalencia de agentes de ITS por orientación sexual declarada, la población heterosexual presenta la frecuencia más alta. Estos resultados fueron similares a un estudio que evaluó la relevancia de infección por VIH en un centro penitenciario en España en año 201222. Es necesario señalar que los resultados en heterosexuales podrían deberse a un "comportamiento homosexual situacional", debido a la contingencia que conduce a conductas homosexuales para generar satisfacción sexual con hombres del mismo recinto, sin protección ${ }^{16,22,23}$.

Una limitación de este estudio es que no se tiene registro de cuántos hombres rechazaron participar en el estudio, ya que los que aceptaron voluntariamente, asistían al Centro de Salud por diversos motivos de salud o control (diabetes mellitus, hipertensos, entre otros), pudiendo este hecho influir en la frecuencia poblacional de la ITS detectada.

La contribución más importante del presente estudio es dar a conocer la realidad de la frecuencia de infección por N. gonorrhoeae y algunos factores de riesgo asociados, en el recinto penitenciario de la zona trifronteriza del norte de Chile, ya que de acuerdo a lo referido por la Fundación Savia "poco se conoce de las ITS, no existen programas de prevención y autocuidado respecto a estas infecciones"23. Los programas educativos de salud sexual y reproductiva podrían ayudar a disminuir las prácticas sexuales de riesgo, tanto dentro del sistema carcelario como en la comunidad, lo que evitaría la transmisión de ITS entre los reclusos y a la población general al cumplir éstos sus condenas y reintegrarse a la comunidad. La 


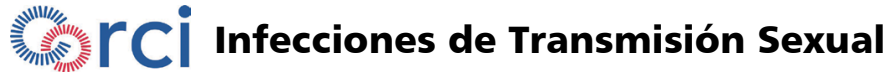

motivación es continuar con investigaciones similares que sean un aporte a la región en la implementación de políticas públicas locales, para la prevención de las ITS en el recinto carcelario.
Agradecimientos. Al personal del Centro de Salud de Gendarmería del CDP Arica. A M. Angélica Martínez Tagle, Q.E.P.D., por su entrega y valiosa contribución en nuestra investigación.

\section{Referencias bibliográficas}

1.- Guía práctica del tratamiento sindrómico de las infecciones de transmisión sexual: una propuesta de tratamiento. Guía de consenso. http://www.medigraphic.com/pdfs/derrevmex/ rmd-2014/rmd141d.pdf

2.- World Health Organization. Sexually transmitted diseases. Fact sheet $\mathrm{N}^{\circ} 110$, actualizado a diciembre del $2015 \mathrm{https}: / /$ communitymedicine4asses.wordpress. com/2015/12/14/who-updates-fact-sheeton-sexually-transmitted-infections-stis-9december-2015/

3.- OMS. Infecciones de transmisión sexual. Nota descriptiva $\mathrm{N}^{\circ} 110$. Agosto 2016. http://www. who.int/mediacentre/factsheets/fs110/es/

4.- MINSAL. Infecciones de transmisión sexual: sífilis y gonorrea. El Vigía 27. 13 (27). 2012. https://es.slideshare.net/Cesfamgarin/vigia-27

5.- MINSAL. Vigilancia de Neisseria gonorrhoeae, Chile 2010-2016. Boletín de Laboratorio y Vigilancia al Día 6 (12). 2016. http://www. ispch.cl/sites/default/files/Boletin\%20GonoFinal.pdf.

6.- Cáceres K. Informe Anual 2016 Situación epidemiológica de gonorrea (CIE 10: A54). Rev Chilena Infectol 2018; 35 (4): 403-12. http://dx.doi.org/10.4067/s071610182018000400403.

7.- Guías para el tratamiento de las infecciones de transmisión sexual. Organización Mundial de la Salud; 2005. http://files.sld.cu/sida/ files/2016/06/manejo-de-its spa.pdf.

8.- Sánchez R R, Alonso P J P, Santabárbara S J. Infecciones de transmisión sexual en hombres internos en prisión: riesgo de desarrollo de nuevas infecciones. Gac Sanit 2016; 30 (3): 208-14. http://dx.doi.org/10.1016/j. gaceta.2016.01.010.

9.- Guerrero G. Mayelis. Conocimientos, actitudes y prácticas sexuales riesgosas ante el VIH/sida en población privada de la libertad. El caso de Cartagena. 2012 Bogotá, Colombia. http://www.bdigital.unal.edu. co/10440/1/597698.2012.pdf.

10.- Informe Final ${ }^{\circ} 33$, de 2012, sobre Auditoría efectuada a los Programas de Prevención del VIH/SIDA, en recintos penitenciarios de Gendarmería de Chile. https://html. gendarmeria.gob.cl/doc/transparencia/ley20285/ doc 2009/auditoria/InformesFinales/33 2012 PROGRAMAS_PREVENCION_VIHSIDA_ EN_RECINTOS_PENITENCIARIOS.pdf.

11.- Gendarmería de Chile. Estadística de población penal, junio 2012. http://www.gendarmeria.gob. $\mathrm{cl} /$ estadisticas pobpenal.jsp.

12.- Garaycochea M C, Pino R, Chávez I, Portilla J L, Miraval M L, Arguedas E, et al. Infecciones de transmisión sexual en mujeres de un establecimiento penitenciario de Lima, Perú. Rev Perú Méd Exp Salud Publica 2013; 30 (3): 423-7. http://www.scielo.org.pe/pdf/rins/v30n3/ a08v30n3.pdf.

13.- Bickell N A, Vermund S H, Holmes M, Safyer S, Burk R D. Human papillomavirus, gonorrhea, sypyllis, and cervical dysplasia in jailed women. Am J Public Health 1991; 81: 1318-20. https://dol.org/10.2105/ AJPH.81.10.1318.

14.- Zotta C, Lavayén S. Galeano G, Gianecini R, Oviedo C, Galarza P. Infección por Neisseria gonorrhoeae y fenotipos de resistencia antimicrobiana. Mar del Plata. 2005-2010. Acta Bioq Clín Latinoam 2014; 48 (4) 475-83. https://www.redalcyc.org/articulo. oa? id=535/53535594010.

15.- Pineda S, Minuzzi G, Gárate L, Giacamann $\mathrm{P}$, Zemelman V. Infecciones de transmisión sexual: Concordancia diagnóstica entre profesionales de atención primaria de la zona norte de Santiago y dermatólogos del Hospital San José. 2016. Rev Chile Dermatol 32 (3). doi: http://dx.doi.org/10.31879/rcderm. v32i3.122.

16.- Vall-Mayans M. Infecciones de transmisión sexual en el medio penitenciario. España: Rev Esp Sanidad Penitenciaria 2013; 15 (1): 1-2. $\mathrm{http} / / /$ scielo.isciii.es/scielo.php?script=sci arttext\&pid=S1575-06202013000100001\&lng= es\&tlng $=\mathrm{es}$.

17.- Sánchez M, Piñol D. Condiciones de vida en los centros de privación de libertad en Chile. http://www.cesc.udechile.cl/docs/CESC_ condiones_centros_privacion.pdf.

18.- García-Guerrero J, Marco A. Sobrepoblación en los centros penitenciarios y su impacto en la salud. Rev. Esp Sanid Penit 2012; 14: 106-13. http://scielo.isciii.es/pdf/sanipe/v14n3/en_06_ revision2.pdf.

19.- Mendoza L A, Arias M, Pedroza M, Micolta P, Ramírez A, Cáceres C, et al. Actividad sexual en adolescencia temprana: problema de salud pública en una ciudad colombiana. Rev Chil Obstet Ginecol 2012; 77: 271-9. https://scielo.conicyt.cl/scielo.php?pid=S0717$75262012000400006 \&$ script $=$ sci arttext\&tlng=en.

20.- INJUV - Ministerio de Desarrollo Social. VIII Encuesta Nacional de la Juventud 2015. http://www.injuv.gob.cl/portal/wp-content/ uploads/2017/03/libro-octava-encuestanacional-de-juventud.pdf.

21.- Bórquez C, Lobato I, Gazmuri P, Hurtado R, Llanqui V, Vivanco M, et al. Prevalencia del virus de la inmunodeficiencia humana, virus de la hepatitis B y Treponema pallidum en reclusos del Centro de Detención Preventiva de Arica, Chile. Rev Chilena Infectol 2017; 34 (5): 453-7. http://dx.doi.org/10.4067/S071610182017000500453.

22.- Marco A, Saiz de la Hoya P, García-Guerrero J y Grupo PREVALHEP. Estudio multicéntrico de prevalencia de infección por el VIH y factores asociados en las prisiones de España. Rev Esp Sanid Penit 2012; 14: 19-27. http:// scielo.isciii.es/pdf/sanipe/v14n1/en 04 original3.pdf.

23.- Fundación Savia. Investigación de monitoreo y valoración de la política pública de prevención y atención integral del VIH y SIDA en población privada de libertad. http:// www.fundacionsavia.cl/media/uploads/docs/ INVESTIGACION_EN_POBLACION PRIVADOS_DE_LIBERTAD_1.pdf. 